\title{
Orbitofrontal Cortex Mediates Outcome Encoding in Pavlovian But Not Instrumental Conditioning
}

\author{
Sean B. Ostlund and Bernard W. Balleine \\ Department of Psychology and the Brain Research Institute, University of California, Los Angeles, California 90095-1563
}

Previous studies have implicated the orbitofrontal cortex (OFC) in outcome encoding. However, it remains unknown whether the OFC is selectively involved in pavlovian stimulus- outcome learning or whether it also contributes to instrumental action- outcome learning. In experiment 1 , we investigated this issue by assessing the effects of bilateral lesions of the OFC on the sensitivity of instrumental lever press performance to a reduction in the incentive value of the training outcome (a test of action-outcome encoding) and to outcome-specific pavlovian-instrumental transfer (a test of stimulus- outcome encoding). We found that post-training lesions of the OFC did not affect instrumental outcome devaluation, but abolished the transfer effect. Interestingly, lesions made before training had no effect on either task. In experiment 2, we explored the involvement of the OFC in updating stimulus- outcome associations after the underlying contingency, or predictive relationship, between these two events has been degraded. Shams displayed clear contingency learning, withholding conditioned responding to a stimulus that no longer reliably predicted its outcome while continuing to respond to a control stimulus that remained a good predictor of a different outcome. In contrast, OFC-lesioned rats stopped responding to both stimuli, regardless of their predictive status. Together, these findings suggest that the OFC supports outcome encoding in pavlovian, but not instrumental conditioning.

Key words: causal learning; contingency; incentive; cognition; motivation; prefrontal cortex; response selection; reward

\section{Introduction}

Outcome encoding plays an important role in the acquisition and expression of pavlovian stimulus-outcome and instrumental action-outcome associations. For instance, pavlovian conditioned approach behavior (Holland and Straub, 1979) and instrumental lever pressing (Adams and Dickinson, 1981; Colwill and Rescorla, 1985) are both sensitive to post-training manipulations of outcome value, indicating that, in each case, performance is mediated by a representation of the training outcome. Furthermore, conditioned stimulus (CS) presentations tend to facilitate instrumental performance on the basis of a shared outcome representation, an effect commonly referred to as outcome-selective pavlovian-instrumental transfer (Kruse et al., 1983). Outcome encoding also appears to be involved in updating previously acquired stimulus-outcome (Delamater, 1995) and action-outcome (Colwill and Rescorla, 1986; Balleine and Dickinson, 1998) associations after a shift in the contingent, or predictive, relationship between these events.

Recent studies using a range of species and experimental techniques have implicated the orbitofrontal cortex (OFC) in outcome encoding (for review, see Holland and Gallagher, 2004; Schoenbaum and Roesch, 2005). For instance, electrophysiological studies in both rats (Schoenbaum et al., 1998) and primates

Received Dec. 15, 2006; revised March 25, 2007; accepted March 28, 2007. This work was supported by National Institute of Mental Health Grant 56446.

Correspondence should be addressed to Sean 0stlund, Department of Psychology, University of California, Los Angeles, Box 951563, Los Angeles, CA 90095-1563. E-mail: sostlund@ucla.edu. D01:10.1523/JNEUROSCI.5443-06.2007

Copyright $\odot 2007$ Society for Neuroscience $\quad$ 0270-6474/07/274819-07\$15.00/0
(Tremblay and Schultz, 2000) have found outcome-related neural activity in the OFC during performance of a go/no-go discrimination. Furthermore, OFC lesions abolish the sensitivity of conditioned approach behavior to post-training outcome devaluation (Gallagher et al., 1999; Pickens et al., 2003, 2005) and disrupt the excitatory influence that pavlovian outcome expectations have on instrumental response selection (McDannald et al., 2005).

In general, evidence that the OFC plays a role in outcome encoding has been obtained using behavioral tasks that are likely to depend primarily on stimulus-outcome learning. Therefore, the contribution of the OFC to goal directed, instrumental performance remains unknown. To explore this issue, experiment 1 assessed the effects of bilateral OFC lesions on the sensitivity of instrumental lever pressing to outcome devaluation and to pavlovian-instrumental transfer. We found that lesions made after initial training had no detectable effect on outcome devaluation performance but abolished outcome-selective transfer. Experiment 2 investigated the role of the OFC in pavlovian contingency learning. We found that, although OFC-lesioned rats displayed normal levels of conditioned approach behavior to a CS that had been paired with food, they were unable to appropriately adjust their performance once the predictive status of that CS was degraded. Together, the findings reported here indicate that the OFC plays a critical role in outcome encoding in pavlovian, but not instrumental conditioning.

\section{Materials and Methods}

Subjects and apparatus

Adult female Long-Evans rats obtained from Harlan Industries (Indianapolis, IN) were used as subjects. Rats were housed in pairs in transpar- 
ent plastic tubs located in a temperature- and humidity-controlled vivarium. A food deprivation regimen was used during behavioral training and testing to maintain the subjects at $\sim 85 \%$ their ad libitum-feeding body weight. The behavioral procedures were performed in 16 identical Med Associates (East Fairfield, VT) behavioral chambers enclosed in sound- and light-attenuating shells. Each chamber was equipped with two retractable levers that could be extended to the left and right of a recessed food magazine. Attached to the food magazine was a pellet dispenser, used to deliver $45 \mathrm{mg}$ grain-based food pellets (Bio-Serv, Frenchtown, NJ), and an infusion pump fitted with a syringe, used to deliver $0.1 \mathrm{ml}$ drops of $20 \%$ sucrose solution. An infrared photobeam crossed the magazine opening, allowing for the detection of head entries. Illumination was provided by a houselight $(3 \mathrm{~W}, 24 \mathrm{~V})$ located on the wall opposite the magazine. Tone $(3 \mathrm{kHz} ; \sim 80 \mathrm{~dB})$ and white noise $(\sim 80 \mathrm{~dB})$ generators, attached to the exterior of each behavioral chamber, were used to present the two auditory CSs. A pair of microcomputers running the Med-PC program (Med Associates) controlled all experimental events and recorded lever presses and magazine entries.

\section{Surgery}

Subjects were provided with unrestricted access to home chow for at least $24 \mathrm{~h}$ before surgery. At the time of surgery, rats were anesthetized with sodium pentobarbital (Nembutal, $50 \mathrm{mg} / \mathrm{kg}$ ) and placed in a stereotaxic frame (Stoelting, Wood Dale, IL). An incision was made in the scalp, the incisor bar was adjusted to level the skull surface, and a small burr hole was drilled into the skull above each target site. Neurotoxic lesions were made by lowering the needle of a $1 \mu \mathrm{l}$ Hamilton syringe into the target site in each hemisphere (all coordinates, relative to bregma: anteroposterior, +3.5 ; mediolateral, \pm 3.2 ; dorsoventral, -4.7 ) and infusing $0.4 \mu \mathrm{l}$ of NMDA $(20 \mu \mathrm{g} / \mu \mathrm{l}$ in PBS) over $4 \mathrm{~min}$. The needle was left in place for an additional $4 \mathrm{~min}$ to allow for further diffusion of the drug. Sham surgeries were conducted in the same manner except that the needle was not lowered and no infusions were made. Subjects were given at least $10 \mathrm{~d}$ to recover from surgery. Rats were handled and returned to the food deprivation regimen during the last $3 \mathrm{~d}$ of the recovery period.

\section{Experiment 1}

The aim of experiment 1 was to investigate the effects of pretraining and post-training lesions of the OFC on instrumental outcome devaluation and pavlovian-instrumental transfer.

Surgery. Subjects received neurotoxic or sham lesions of the OFC either $10 \mathrm{~d}$ before (OFC, $n=8$; sham, $n=8$ ) or $2 \mathrm{~d}$ after (OFC, $n=8$; sham, $n=8$ ) behavioral training.

Training. Training began with eight daily sessions of pavlovian conditioning in which each of two auditory CSs (tone and white noise) was paired with a different outcome (pellets and sucrose). Half of the rats in each group were given tone-pellet and noise-sucrose pairings and half were given the opposite stimulus-outcome relationships. Each CS was presented for $2 \mathrm{~min}$, during which the corresponding outcome was delivered on a random time $30 \mathrm{~s}$ schedule. Each CS was presented four times per session using a pseudorandom trial order and a variable intertrial interval (ITI; mean, $5 \mathrm{~min}$ ).

The rats then received $11 \mathrm{~d}$ of instrumental conditioning in which two responses (left and right lever presses) were trained with different outcomes (pellets and sucrose) in separate daily sessions. The order of training sessions was alternated over days. Half of the subjects in each group were given left lever-pellet and right lever-sucrose pairings and half were given the opposite response-outcome relationships (counterbalanced with pavlovian stimulus-outcome relationships). Each session was terminated after 15 outcomes were earned or $30 \mathrm{~min}$ had elapsed, whichever came first. For the first $2 \mathrm{~d}$ of instrumental training, lever pressing was continuously reinforced (CRF), such that each action resulted in an outcome delivery $(p=1.0)$. The probability of the outcome given a response was then gradually shifted over days using increasing random ratio (RR) schedules: an RR-5 schedule ( $p=0.2)$ was used on days $3-5$, an RR-10 schedule $(p=0.1)$ was used on days $6-8$, and an RR-20 schedule $(p=$ $0.05)$ was used on days $9-11$.

Testing. Subjects were first given two sessions of outcome devaluation testing. Before each test session, one of the two training outcomes was selectively devalued through specific satiety (Balleine and Dickinson, 1998). Subjects were given $1 \mathrm{~h}$ of unrestricted access to either pellets or sucrose solution in their home cage immediately before being returned to the experimental chamber for a 5 min choice extinction test. Both levers were inserted into the box throughout the session but no outcomes were delivered. Subjects were given a second test $48 \mathrm{~h}$ later with the other outcome devalued. Test order was counterbalanced with surgical group and training contingencies.

Two days later, subjects were returned to the behavioral chamber for a single pavlovian-instrumental transfer test. Once again, both levers were inserted into the box throughout the session but no outcomes were delivered. Responding was extinguished on both levers for $8 \mathrm{~min}$ to establish a low rate of baseline performance. Each CS was presented four times over the next $40 \mathrm{~min}$ in the following order: noise-tone-tone-noise-tonenoise-noise-tone. Stimulus presentations lasted $2 \mathrm{~min}$ and were separated by a $3 \mathrm{~min}$ fixed ITI.

\section{Experiment 2}

Pavlovian outcome expectations are not only capable of eliciting reflexive preparatory behavior (e.g., conditioned magazine approach) and guiding response selection (e.g., pavlovian-instrumental transfer), they have also been shown to play a critical role in updating stimulus-outcome associations in response to degradation of the contingency between these two events (Delamater, 1995). Specifically, it is possible to selectively weaken the predictive status of a previously trained CS by delivering its outcome with the same probability in its presence as in its absence. Thus, although the CS continues to be paired with its outcome, it is no more reliable at signaling that outcome than any other cue in the experimental context. Importantly, this treatment does not impact conditioned responding to another CS that predicts a different outcome, demonstrating that subjects are able to adaptively adjust their performance to the appropriate stimulus based on its anticipated outcome. We assessed the effect of OFC lesions on stimulus-outcome contingency degradation in experiment 2 .

Training. Intact subjects were given eight daily sessions of pavlovian conditioning using a procedure similar to that described in experiment 1 . As before, half the subjects were given tone-pellet and noise-sucrose pairings and half were given the opposite stimulus-outcome relationships. Unlike experiment 1, however, each CS presentation lasted $20 \mathrm{~s}$ and was immediately followed by the delivery of the appropriate outcome with a probability of 0.5 . Each stimulus was presented eight times per session using a pseudorandom trial order and a variable ITI (mean, 4 $\min )$.

Surgery. Two days after training, subjects were given neurotoxic $(n=$ 7) or sham $(n=8)$ lesions of the OFC.

Pavlovian contingency degradation. After the surgical recovery period, subjects were given 10 daily sessions of pavlovian contingency degradation training. Sessions were otherwise identical to those used during initial pavlovian training except that the predictive relationship between one CS and its outcome was degraded by delivering that outcome (either pellet or sucrose, counterbalanced) noncontingently with a probability of 0.5 in each $20 \mathrm{~s}$ period during the ITI. This procedure ensured that, although both stimuli continued to be paired with their respective outcomes with the same probability ( $p=0.5$ ), one CS no longer served as a reliable predictor of its specific outcome.

Behavioral measures. Magazine entries were recorded during CS presentations and during periods of equal length immediately preceding each CS. Unless stated otherwise, the tendency of a CS to evoke magazine approach behavior was quantified by subtracting the entry rate during pre-CS periods from that observed during CS periods (i.e., CS - preCS). Lever presses were recorded throughout each session of instrumental training and testing.

Histology. After behavioral testing, the rats received a lethal overdose of sodium pentobarbital and were perfused transcardially with $0.9 \%$ saline followed by $10 \%$ buffered formalin solution. Their brains were then extracted and postfixed in a $25 \%$ sucrose-formalin solution. After several days, the brains were frozen and $50 \mu \mathrm{m}$ coronal sections of the frontal cortex were collected on glass slides. The sections were stained with thionin and examined with a microscope to assess the placement and extent 
of neuronal damage through comparison with control sections from the sham group and a stereotaxic atlas.

\section{Results}

\section{Histological verification of OFC lesions}

Figure 1 presents the results of the histological analysis. All lesioned subjects were found to have substantial bilateral neuronal damage to the OFC. The extent and location OFC damage was similar across subjects and was primarily restricted to the lateral and ventrolateral orbital regions, but extended into the deep layers of the overlying motor cortex and agranular insular cortex in some subjects.

\section{OFC lesions do not affect the acquisition or performance of conditioned approach or instrumental lever pressing} Pavlovian training produced robust conditioned approach behavior in all groups. Figure $2 \mathrm{~A}$ displays the mean number of conditioned magazine entries (CS minus pre-CS) performed per minute across successive two-session blocks for the groups that received sham or OFC lesions before training and for the unoperated group (Unop) ( $n=16$; i.e., those rats that would undergo post-training surgery). A mixed ANOVA using group (sham, OFC, and unoperated) and block (1-4) as factors found a main effect of block $\left(F_{(3,87)}=26.30 ; p<0.001\right)$, but found no evidence of an effect of group or a session by group interaction ( $F$ values $<1$ ). The groups also acquired lever pressing at similar rates during the instrumental training phase of the experiment. Figure $2 B$ (bottom) presents the mean number of presses performed per minute across sessions, blocked by the training contingency that was in place (i.e., CRF, RR-5, RR-10, and RR-20) (see Materials and Methods). A group by block ANOVA found a main effect of block $\left(F_{(3,87)}=153.75 ; p<0.001\right)$, but found no effect of group $(F<1)$ or block by group interaction $\left(F_{(6,87)}=1.20 ; p>0.30\right)$. Thus, the OFC does not appear to be essential for the acquisition or performance of either conditioned approach responses or instrumental lever pressing.

\section{OFC lesions do not affect instrumental outcome devaluation}

After the post-training surgical recovery period, we assessed the sensitivity of each group's instrumental performance to a reversible reduction in outcome value across two tests (see Materials and Methods). The results of these tests were combined after a preliminary analysis failed to detect a significant effect of test or a test by response interaction $(F$ values $<1)$. The rates of responding (presses per minute) on the action that had earned the devalued outcome (Devalued) and on the other action (Other) are presented in Figure 3, plotted separately for each group. All groups displayed a clear and selective suppression in the performance of the action that, in training, delivered the devalued outcome. A mixed ANOVA using time of surgery (presurgery and postsurgery), lesion type (sham and OFC) and devaluation (Devalued and Other) as factors was conducted on these data, resulting in a significant main effect of devaluation $\left(F_{(1,28)}=36.22 ; p<\right.$ $0.001)$, but not of time of surgery or of lesion $(F$ values $<1)$. Furthermore, none of the interactions between these factors reached significance (largest $F_{(1,28)}=1.54 ; p>0.20$ ), indicating that the influence of outcome value over instrumental performance did not reliably differ across groups.

Post-training OFC lesions disrupt outcome selective transfer After outcome devaluation testing, we assessed the impact of OFC damage on the influence of the pavlovian stimulus-outcome associations on instrumental response selection. Figure 4
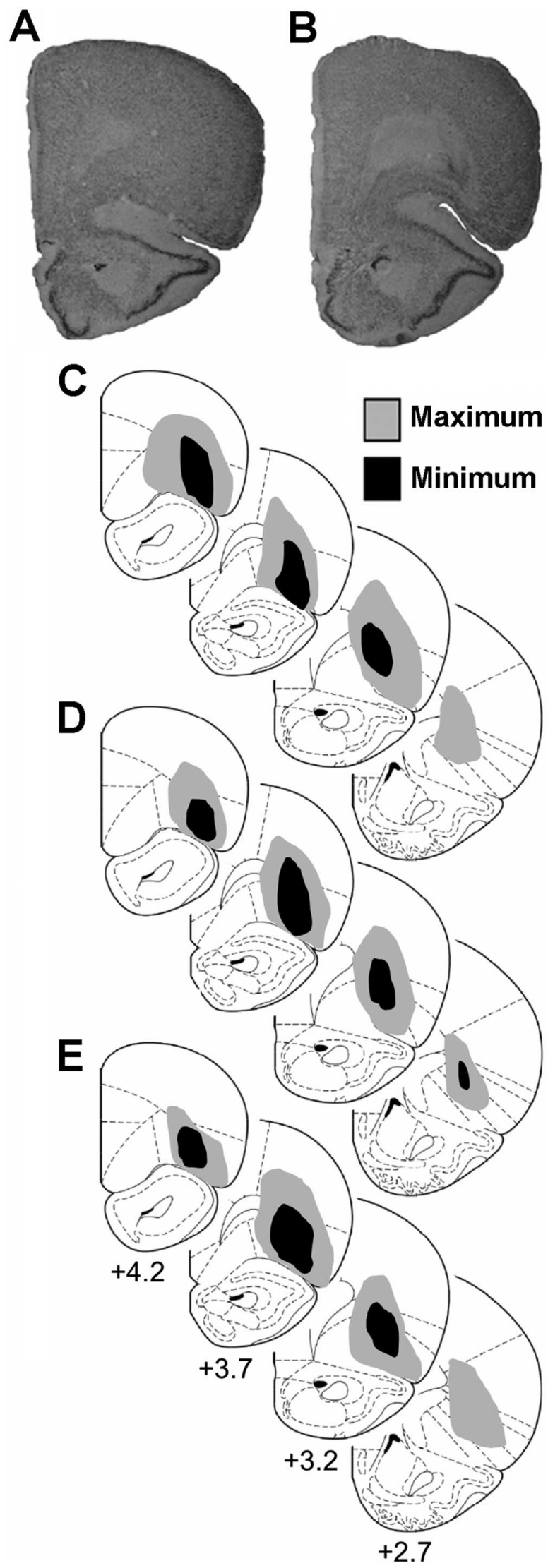

Figure 1. Histological results. $A, B$, Photomicrographs of representative sham $(\boldsymbol{A})$ and $\mathrm{OFC}$ lesions $(\boldsymbol{B})$. $\boldsymbol{C}-\boldsymbol{E}$, Schematic representation of minimum and maximum extent of OFC damage in each of the three lesion groups. $C$ and $\boldsymbol{D}$ refer, respectively, to groups pre- $0 \mathrm{FC}$ and post-OFC in Experiment 1.E refers to the lesioned group in Experiment 2. 


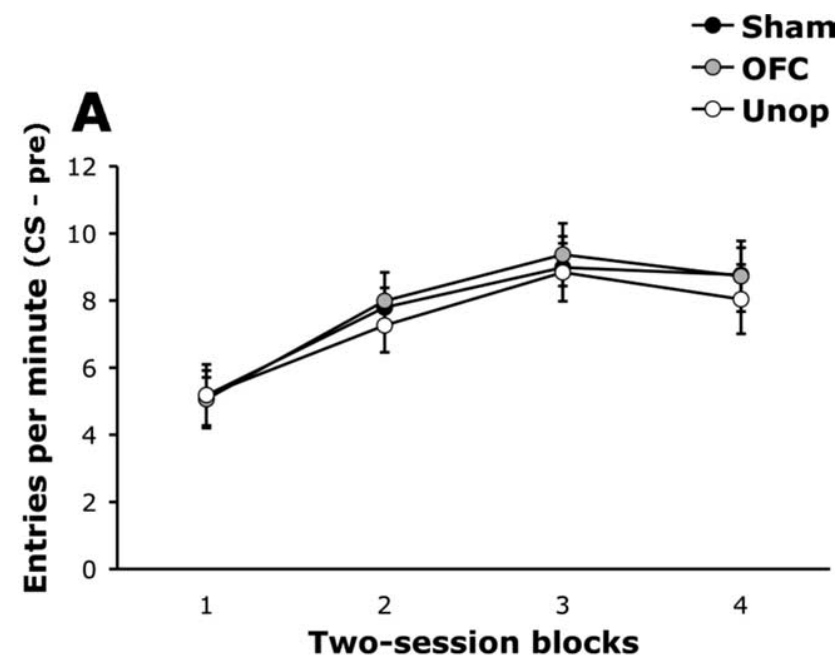

B

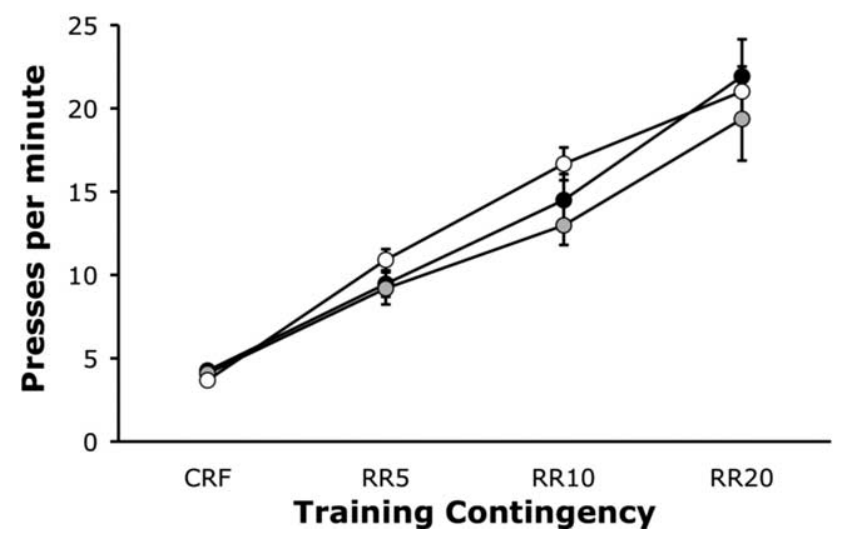

Figure 2. Effect of OFC lesions on the acquisition of pavlovian conditioned approach and instrumental lever pressing. $\boldsymbol{A}$, Mean number of conditioned magazine entries performed per minute ( \pm SEM) across successive two-session blocks of pavlovian training, plotted as the difference in responding during $C S$ and pre-CS periods (CS minus pre-CS). $\boldsymbol{B}$, Mean lever presses performed per minute ( \pm SEM) across sessions of instrumental training, blocked according to training contingency.

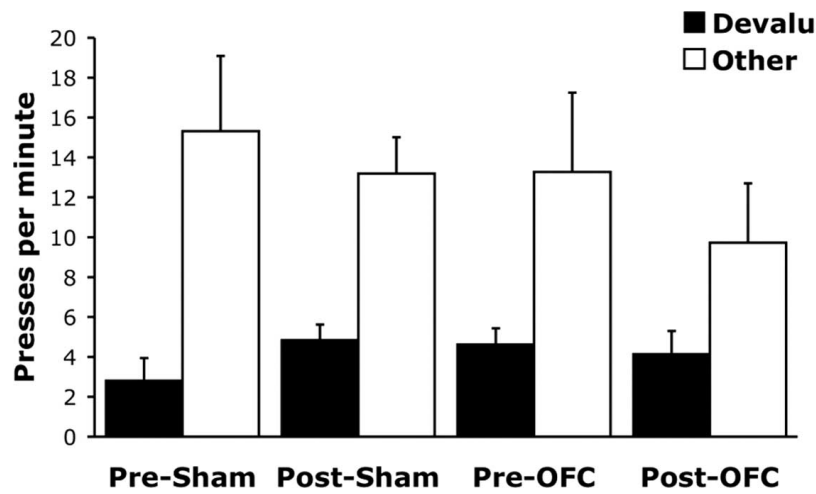

Figure 3. Effect of OFC lesions on sensitivity of instrumental performance to outcome devaluation. Mean lever presses per minute ( + SEM) in the extinction test. The response paired with the devalued outcome (Devalued) and the response paired with the other outcome (0ther) are plotted separately.

presents the rate of responding (mean presses per minute) at test for each group, collapsed across levers, during a period when no CS was presented (Pre-CS), when the CS that predicted the same outcome as the response (Same) was presented, and when the CS

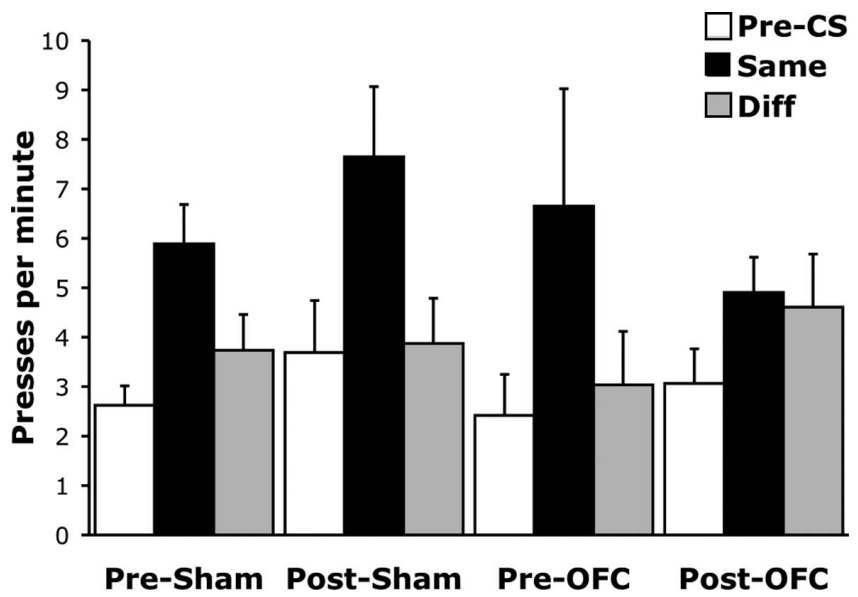

Figure 4. Effect of OFC lesions on pavlovian-instrumental transfer. Mean lever presses per minute (+SEM) during the pre-CS period (Pre-CS), the CS that predicted the same outcome as the response (Same), and the CS that predicted a different outcome (Diff).

that predicted a different outcome from the response (Diff) was presented. These data were again analyzed using a mixed ANOVA in which time of surgery (presurgery and postsurgery), lesion type (sham and OFC) and CS identity (Pre-CS, Same, and Diff) served as factors. The ANOVA detected a significant main effect of CS identity $\left(F_{(2,56)}=21.90 ; p<0.001\right)$, but found no effect of time of surgery $\left(F_{(1,28)}=1.02 ; p>0.30\right)$ or of lesion $(F<$ $1)$. Furthermore, none of the two-factor interactions reached significance $(F$ values $<1)$. However, this analysis did reveal a significant time of surgery by lesion by CS identity three-way interaction $\left(F_{2,56)}=3.16 ; p<0.05\right)$, indicating that influence of the $\mathrm{CSs}$ on response selection varied across groups. The source of this interaction was investigated further by conducting separate time of surgery by CS identity ANOVAs for each lesion condition. For shams, the analysis resulted in a significant effect of CS identity $\left(F_{(2,28)}=9.70 ; p=0.001\right)$, but did not of find an effect of time of surgery $\left(F_{(1,14)}=1.28 ; p>0.25\right)$ or a CS identity by time of surgery interaction $(F<1)$. Furthermore, analysis of their performance across CS identity found that responding was elevated during the CS that predicted the same outcome as the action relative both to the pre-CS period $\left(F_{(1,15)}=16.93 ; p=0.001\right)$ and to the CS that predicted a different outcome $\left(F_{(1,15)}=9.25 ; p<\right.$ $0.01)$. Their performance did not differ between periods Pre-CS and Diff $(F<1)$. For OFC-lesioned rats, although the ANOVA did not detect an effect of time of surgery $(F<1)$, both the effect of CS identity $\left(F_{(2,28)}=14.17 ; p<0.001\right)$ and the CS identity by time of surgery interaction $\left(F_{(2,28)}=12.13 ; p<0.05\right)$ reached significance. Additional analysis revealed that the pre-OFC group responded differently across CS periods $\left(F_{(2,14)}=27.89 ; p<\right.$ 0.001), performing more responses during the Same CS than during the pre-CS period $\left(F_{(1,7)}=56.78 ; p<0.001\right)$ or the Diff CS $\left(F_{(1,7)}=20.07 ; p<0.01\right)$; performance did not differ reliably between the pre-CS and Diff CS periods $\left(F_{(1,7)}=2.85 ; p>0.10\right)$. In striking contrast, no effect of CS identity was observed for the post-OFC group $\left(F_{(2,14)}=1.96 ; p>0.15\right)$, indicating that the facilitatory influence of pavlovian learning over instrumental performance was abolished by OFC lesions made after training.

OFC lesions disrupt the outcome selectivity of pavlovian contingency learning

Contingency degradation has been found to reduce the strength of specific stimulus-outcome associations in pavlovian condi- 

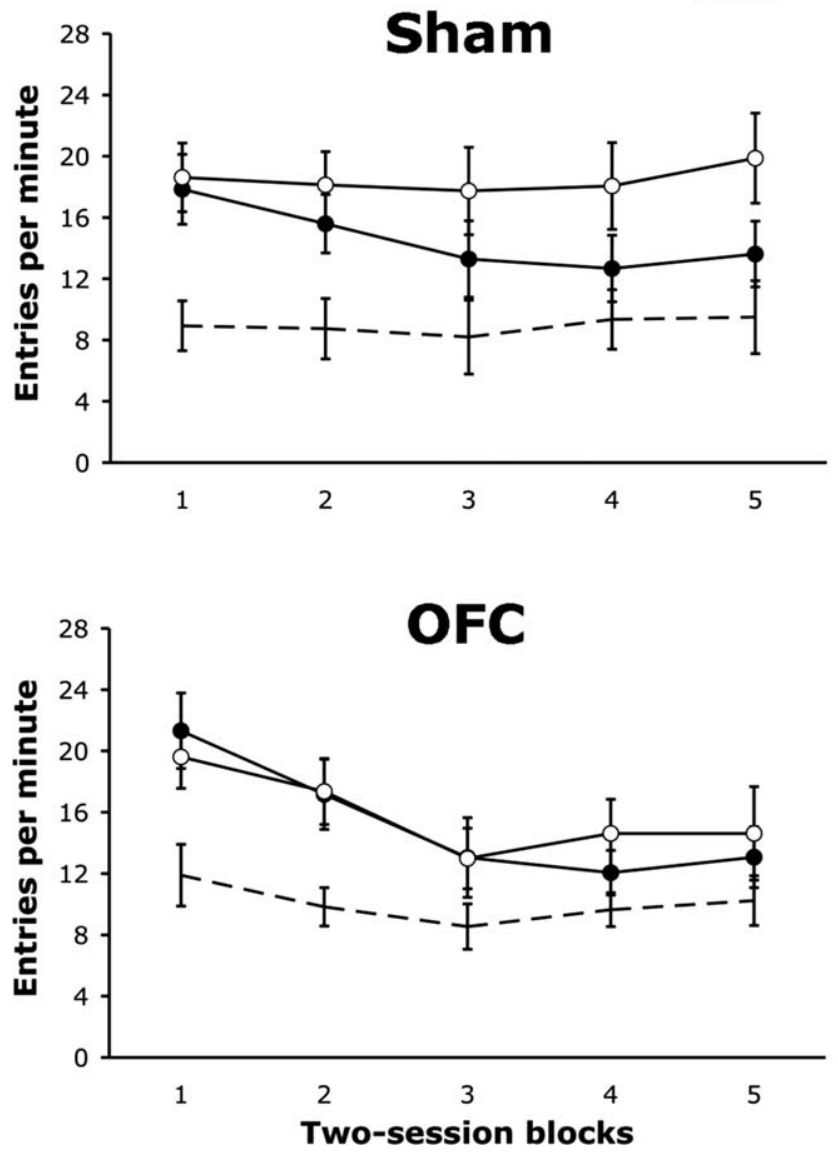

Figure 5. Effect of OFC lesions on pavlovian contingency degradation. Mean number of magazine entries performed per minute ( \pm SEM) over two-session blocks of contingency degradation training, plotted separately for the pre-CS period (Pre-CS), the CS paired with the noncontingent outcome (Degraded), and the other CS (0ther). The data are presented separately for the sham (top) and OFC (bottom) groups.

tioning (Delamater, 1995). We assessed the effect of OFC lesions on sensitivity to changes in the pavlovian contingency in experiment 2 using a within-subjects design in which two stimulusoutcome associations were trained, but only one was degraded. In phase 1, two stimulus-outcome associations were established using a tone and white noise as the CSs and pellets and sucrose as the outcomes. One subject from the sham group failed to acquire conditioned responding to both CSs and was therefore excluded from the data analysis $(n=7)$. The remaining subjects acquired robust rates of responding to both CSs, such that by the last session of training subjects averaged 17.92 (SEM, 1.20) entries per minute during the CSs and 5.40 entries per minute (SEM, $0.55)$ during the pre-CS period. Group assignments for posttraining surgery were matched using a difference score (CS minus pre-CS). On this measure, the mean number of conditioned magazine entries performed per minute in the OFC group (12.35; SEM, 1.02) did not differ significantly from that in the sham group (12.70; SEM, 2.03) $(F<1)$ before surgery.

After surgery, we assessed the impact of OFC damage on pavlovian contingency learning. The data from this phase of the experiment are presented in Figure 5, which shows the rate of magazine approach behavior plotted across successive twosession blocks, for the sham group (top) and for the OFC group (bottom). Responding is presented separately for each CS (Degraded and Other) and for the average pre-CS period. It is clear that the two groups responded differently during contingency degradation training. A mixed ANOVA using group (sham and OFC), CS identity (Pre-CS, Degraded, and Other) and block (1-5) as factors resulted in a significant main effect of block $\left(F_{(4,48)}=3.35 ; p<0.05\right)$ and CS identity $\left(F_{(2,24)}=50.77 ; p<\right.$ $0.001)$, but found no effect of group $(F<1)$. Importantly, a significant group by CS identity interaction $\left(F_{(2,24)}=3.44 ; p<\right.$ $0.05)$ was observed, although the block by group $\left(F_{(4,48)}=1.03\right.$; $p=0.40)$ and block by group by CS identity $(F<1)$ interactions were not significant. Additional analysis of the group by CS identity interaction was conducted to assess the source of this interaction and found that the groups displayed different patterns of responding to the two CSs. A CS identity (Degraded vs Other) by block ANOVA was performed separately on each group's data. For the sham group, this analysis resulted a significant effect of CS identity $\left(F_{(1,6)}=10.39 ; p<0.05\right)$ and a marginal effect of block $\left(F_{(4,24)}=2.29 ; p=0.09\right)$. Importantly, the ANOVA found a significant CS by block interaction $\left(F_{(4,24)}=4.85 ; p<0.01\right)$, indicating that the sham group selectively suppressed their performance to the degraded CS over days, relative to the other CS. In contrast, the OFC group showed a general decrease in responding to both stimuli. An ANOVA performed on their data found a significant main effect of block $\left(F_{(4,24)}=3.00 ; p<0.05\right)$, but found no effect of CS identity $(F<1)$ nor a CS identity by block interaction $\left(F_{(4,24)}=2.11 ; p>0.10\right)$.

\section{Discussion}

Lesions of the OFC had no effect on the sensitivity of instrumental performance to a reduction in outcome value, but were effective in disrupting the influence of pavlovian outcome expectancies over instrumental response selection. Furthermore, although OFC lesions left intact the performance of previously acquired conditioned approach behavior, they were found to disrupt the rats' ability to appropriately adjust their performance to meet a reduction in the underlying pavlovian contingency. These findings provide new clues regarding the role of the OFC in predictive learning.

Several previous studies have shown that OFC lesions disrupt the control that expected outcome value exerts on previously acquired responses (Gallagher et al., 1999; Izquierdo et al., 2004; Pickens et al., 2003, 2005). In each of these studies, however, performance of the target response was likely to have depended, at least in part, on pavlovian learning (Roberts, 2006), supporting the view that the OFC plays an important role in stimulus-outcome encoding (Jones and Mishkin, 1972). However, such findings are also readily explained if the OFC in required for either response inhibition (Dias et al., 1997) or encoding the incentive properties of rewarding outcomes (Rolls, 2006). To test these alternative accounts and explore the possibility that the OFC plays a broader role in outcome encoding, we assessed whether OFC lesions would similarly disrupt the sensitivity of instrumental lever pressing to outcome devaluation, a task that has been extensively studied and shown to depend primarily on actionoutcome learning (for review, see Balleine and Dickinson, 1998; Balleine, 2005). In experiment 1 , we found that neither pretraining nor post-training lesions had an effect on instrumental outcome devaluation, demonstrating that these rats were unimpaired in inhibiting a response based on a reduction in the current incentive value of its associated outcome. We then assessed the effect of OFC lesions on the outcome-specific form of pavlovian-instrumental transfer to investigate whether this struc- 
ture is preferentially involved in using pavlovian outcome expectancies to guide behavior. We found that lesions made after, but not before, training were effective in abolishing transfer. Because this deficit involved a failure to increase performance, it is clearly inconsistent with the response inhibition account of OFC function. Furthermore, because transfer has been shown to be insensitive to devaluation of the outcome associated with the CS (Colwill and Rescorla, 1990; Rescorla, 1994; Holland, 2004), and appears to depend primarily on the predictive status of that CS (Delamater, 1995), it is hard to imagine how a purely incentivemotivational view of OFC function could account for this deficit. It is also important to recognize that the use of instrumental outcome devaluation and pavlovian-instrumental transfer tests in the current study allowed us to assess the status of actionoutcome and stimulus-outcome learning, respectively, using a common lever-press response. As a consequence, the different effects of OFC lesions across these tests reflect changes in the distinct cognitive processes engaged by these two tasks rather than an effect on motor performance per se.

The differential effects of pretraining and post-training lesions reported here are compatible with a mnemonic account of OFC function. Although the area of the OFC targeted in our study does not seem to be required for stimulus-outcome encoding, it does appear to be recruited to support such learning if it is intact during initial training. Indeed, although the effect of OFC lesions on pavlovian contingency degradation in experiment 2 might be viewed as a deficit in encoding new stimulus-outcome information, it is worth noting that these lesions were made after preliminary stimulus-outcome training, providing an opportunity for the OFC to become engaged. However, although this dissociation between pretraining and post-training lesions nicely illustrates the nature of the contribution of the OFC to stimulusoutcome encoding, it has been shown that large pretraining lesions (encompassing the medial orbital cortex and both dorsal and ventral agranular cortices) are capable of disrupting pavlovian outcome devaluation performance (Gallagher et al., 1999) and the differential outcomes effect (McDannald et al., 2005), which, like transfer, depends on the integration of pavlovian and instrumental learning (Trapold and Overmier, 1972), suggesting that more extensive lesions made before training would similarly impair transfer. Alternatively, it is possible that, in the absence of a functional OFC, transfer performance can be supported by an alternative behavioral strategy (and corresponding neural circuitry). Additional research will be needed to address this issue.

Several studies have shown that OFC lesions disrupt reversal learning (Jones and Mishkin, 1972; Dias et al., 1997; Schoenbaum et al., 2002; Chudasama and Robbins, 2003), suggesting that this structure may play an important role in the flexible modification of previously acquired stimulus-outcome associations. However, correct performance on this task is not diagnostic of stimulus-outcome encoding because, in fact, there are alternative associative solutions to this problem (Roberts, 2006). In the current study, we assessed the effect of OFC lesions on a pavlovian contingency learning task known to involve updating specific stimulus-outcome associations (Delamater, 1995) (see Materials and Methods). In the current study, we found that sham-lesioned rats appropriately reduced their conditioned performance to a CS that no longer served as a reliable signal of one specific outcome, but maintained high levels of responding to a control CS that continued to be a unique predictor of another outcome. In contrast, OFC-lesioned rats displayed a nonspecific reduction in responding to both stimuli. To interpret this impairment, it is necessary first to note that contemporary associative learning models
(Rescorla and Wagner, 1972) typically explain the contingency degradation effect by assuming that the CS competes with other stimuli, most notably contextual cues, for association with the noncontiguously delivered outcome. According to this view, the selectivity of the sham group's performance demonstrates their ability to generate and contrast highly specific outcome predictions based on the discrete CSs presented during training. The general decrement displayed by the OFC group, therefore, suggests that the specificity of outcome encoding may have been disrupted in these rats, leaving cues to compete based on outcome predictions composed largely of common features. However, an alternative interpretation of these results is that, by undermining the pavlovian learning system that normally mediates the control of conditioned approach, OFC lesions simply abolish the sensitivity of the approach response to manipulations of stimulus-outcome contingency. Consequently, conditioned approach may have fallen under the control of an intact instrumental learning system. On this account, the absence of an explicit action-outcome contingency between the approach response and the delivery of one or other outcome in the current experiment would have resulted in a reduction in instrumental control and, therefore, in the gradual extinction of performance over sessions. In line with this account, Chudasama and Robbins (2003) reported that OFC lesions rendered the performance of a conditioned approach response abnormally sensitive to the introduction of an instrumental omission contingency.

Whichever of these accounts turns out best to characterize the effects of OFC lesions, it is clear that such findings differ dramatically from those produced by lesions made more medially in the prelimbic cortex (PL). Balleine and Dickinson (1998) were the first to report that, in contrast to the OFC lesions in the current study, cell body lesions restricted to the PL abolished the effect of outcome devaluation on instrumental performance. More recently, these effects were replicated and extended to show that, whereas PL lesions abolished outcome devaluation, they left intact pavlovian-instrumental transfer (Corbit and Balleine, 2003) and instrumental reinstatement (Ostlund and Balleine, 2005), suggesting that the PL plays little if any role in acquiring stimulusoutcome or stimulus-response associations. Furthermore, whereas the OFC appears to play a enduring role in pavlovian learning, it has been shown that the PL plays a stage-dependent role in instrumental learning, such that it necessary for the acquisition, but not expression of action-outcome associations (Ostlund and Balleine, 2005). These findings indicate a division of function within the frontal cortex with the PL supporting instrumental processes and the OFC supporting pavlovian processes. In this context, it is interesting to consider the role of the amygdala, particularly the basolateral complex (BLA), which numerous studies have implicated in both instrumental and pavlovian learning (for review, see Balleine and Killcross, 2006). BLA lesions have been shown to disrupt both the impact of outcome devaluation on conditioned approach behavior (Hatfield et al., 1996; Blundell et al., 2003) and the transfer effect (Blundell et al., 2001; Corbit and Balleine, 2005). However, the BLA also appears to be important for encoding action-outcome contingencies and mediating the influence of outcome value on instrumental performance (Balleine et al., 2003; Corbit and Balleine, 2005; Wang et al., 2005), suggesting that it may play a more general role in outcome encoding. Interestingly, the PL and OFC share rich reciprocal projections with somewhat distinct regions of the BLA (McDonald, 1991; McDonald et al., 1996). This differential connectivity may signal heterogeneity of function in associative learning within the BLA. Establishing whether this is true and, if 
so, how these regions of the BLA interact with the prefrontal cortex to support outcome encoding generally, will provide a fruitful avenue for future research.

\section{References}

Adams CD, Dickinson A (1981) Instrumental responding following reinforcer devaluation. Q J Exp Psychol B 33:109-122.

Balleine BW (2005) Neural bases of food-seeking: affect, arousal and reward in corticostriatolimbic circuits. Physiol Behav 86:717-730.

Balleine BW, Dickinson A (1998) Goal-directed instrumental action: contingency and incentive learning and their cortical substrates. Neuropharmacology 37:407-419.

Balleine BW, Killcross S (2006) Parallel incentive processing: an integrated view of amygdala function. Trends Neurosci 29:272-279.

Balleine BW, Killcross AS, Dickinson A (2003) The effect of lesions of basolateral amygdala on instrumental conditioning. J Neurosci 23:666-675.

Blundell P, Hall G, Killcross S (2001) Lesions of the basolateral amygdala disrupt selective aspects of reinforcer representation in rats. J Neurosci 21:9018-9026.

Blundell P, Hall G, Killcross S (2003) Preserved sensitivity to outcome value after lesions of the basolateral amygdala. J Neurosci 23:7702-7709.

Chudasama Y, Robbins TW (2003) Dissociable contributions of the orbitofrontal and infralimbic cortex to pavlovian autoshaping and discrimination reversal learning: further evidence for the functional heterogeneity of the rodent frontal cortex. J Neurosci 23:8771-8780.

Colwill RM, Rescorla RA (1985) Postconditioning devaluation of a reinforcer affects instrumental responding. J Exp Psychol Anim Behav Process 11:120-132.

Colwill RM, Rescorla RA (1986) Associative structures in instrumental conditioning. In: The psychology of learning and memory, Vol 20 (Bower GH, ed), pp 55-104. New York: Academic.

Colwill RM, Rescorla RA (1990) Effect of reinforcer devaluation on discriminative control of instrumental behavior. J Exp Psychol Anim Behav Process 16:40-47.

Corbit LH, Balleine BW (2003) The role of prelimbic cortex in instrumental conditioning. Behav Brain Res 146:145-157.

Corbit LH, Balleine BW (2005) Double dissociation of basolateral and central amygdala lesions on the general and outcome-specific forms of pavlovian-instrumental transfer. J Neurosci 25:962-970.

Delamater AR (1995) Outcome-selective effects of intertrial reinforcement in a pavlovian appetitive conditioning paradigm with rat. Anim Learn Behav 23:31-39.

Dias R, Robbins TW, Roberts AC (1997) Dissociable forms of inhibitory control within prefrontal cortex with an analog of the Wisconsin Card Sort Test: restriction to novel situations and independence from "online" processing. J Neurosci 17:9285-9297.

Gallagher M, McMahan RW, Schoenbaum G (1999) Orbitofrontal cortex and representation of incentive value in associative learning. J Neurosci 19:6610-6614

Hatfield T, Han JS, Conley M, Gallagher M, Holland P (1996) Neurotoxic lesions of basolateral, but not central, amygdala interfere with pavlovian second-order conditioning and reinforcer devaluation effects. J Neurosci 16:5256-5265.

Holland PC (2004) Relations between pavlovian-instrumental transfer and reinforcer devaluation. J Exp Psychol Anim Behav Process 30:104-117.

Holland PC, Gallagher M (2004) Amygdala-frontal interactions and reward expectancy. Curr Opin Neurobiol 14:148-155.
Holland PC, Straub JJ (1979) Differential effects of two ways of devaluing the unconditioned stimulus after pavlovian appetitive conditioning. J Exp Psychol Anim Behav Process 5:65-78.

Izquierdo A, Suda RK, Murray EA (2004) Bilateral orbital prefrontal cortex lesions in rhesus monkeys disrupt choices guided by both reward value and reward contingency. J Neurosci 24:7540-7548.

Jones B, Mishkin M (1972) Limbic lesions and the problem of stimulusreinforcement associations. Exp Neurol 36:362-377.

Kruse JM, Overmier JB, Konz WA, Rokke E (1983) Pavlovian conditioned stimulus effects on instrumental choice behavior are reinforcer specific. Learn Motiv 14:165-181.

McDannald MA, Saddoris MP, Gallagher M, Holland PC (2005) Lesions of orbitofrontal cortex impair rats' differential outcome expectancy learning but not conditioned stimulus-potentiated feeding. J Neurosci 25:4626-4632.

McDonald AJ (1991) Organization of amygdaloid projections to the prefrontal cortex and associated striatum in the rat. Neuroscience 44:1-14.

McDonald AJ, Mascagni F, Guo L (1996) Projections of the medial and lateral prefrontal cortices to the amygdala: a Phaseolus vulgaris leucoagglutinin study in the rat. Neuroscience 71:55-75.

Ostlund SB, Balleine BW (2005) Lesions of medial prefrontal cortex disrupt the acquisition but not the expression of goal-directed learning. J Neurosci 25:7763-7770.

Pickens CL, Saddoris MP, Setlow B, Gallagher M, Holland PC, Schoenbaum G (2003) Different roles for orbitofrontal cortex and basolateral amygdala in a reinforcer devaluation task. J Neurosci 23:11078-11084.

Pickens CL, Saddoris MP, Gallagher M, Holland PC (2005) Orbitofrontal lesions impair use of cue-outcome associations in a devaluation task Behav Neurosci 119:317-322.

Rescorla RA (1994) Transfer of instrumental control mediated by a devalued outcome. Anim Learn Behav 22:27-33.

Rescorla RA, Wagner AR (1972) A theory of pavlovian conditioning and the effectiveness of reinforcement and non-reinforcement. In: Classical conditioning. 2: current research and theory (Black AH, Prokasy WF, eds), pp 64-69. New York: Appleton-Century-Crofts.

Roberts AC (2006) Primate orbitofrontal cortex and adaptive behaviour. Trends Cogn Sci 10:83-90.

Rolls ET (2006) Brain mechanism underlying flavour and appetite. Philos Trans R Soc Lond B Biol Sci 361:1123-1136.

Schoenbaum G, Roesch M (2005) Orbitofrontal cortex, associative learning, and expectancies. Neuron 47:633-636.

Schoenbaum G, Chiba AA, Gallagher M (1998) Orbitofrontal cortex and basolateral amygdala encode expected outcomes during learning. Nat Neurosci 1:155-159.

Schoenbaum G, Nugent S, Saddoris MP, Setlow B (2002) Orbitofrontal lesions in rats impair reversal but not acquisition of go, no-go odor discriminations. NeuroReport 13:885-890.

Trapold MA, Overmier JB (1972) The second learning process in instrumental learning. In: Classical conditioning 2: current research and theory (Black AA, Prokasy WF, eds), pp 427-452. New York: Appleton-Century-Crofts.

Tremblay L, Schultz W (2000) Reward-related neuronal activity during gonogo task performance in primate orbitofrontal cortex. J Neurophysiol 83:1864-1876.

Wang SH, Ostlund SB, Nader K, Balleine BW (2005) Consolidation and reconsolidation of incentive learning in the amygdala. J Neurosci 26:830 835. 